\title{
Indistinguishable Photons from Separated Silicon-Vacancy Centers in Diamond
}

\author{
A. Sipahigil, ${ }^{1, *}$ K. D. Jahnke, ${ }^{2}$ L. J. Rogers, ${ }^{2}$ T. Teraji, ${ }^{3}$ J. Isoya,${ }^{4}$ A. S. Zibrov, ${ }^{1}$ F. Jelezko, ${ }^{2}$ and M. D. Lukin ${ }^{1}$ \\ ${ }^{1}$ Department of Physics, Harvard University, 17 Oxford Street, Cambridge, Massachusetts 02138, USA \\ ${ }^{2}$ Institute for Quantum Optics, University Ulm, Albert-Einstein-Allee 11, 89081 Ulm, Germany \\ ${ }^{3}$ National Institute for Materials Science, 1-1 Namiki, Tsukuba, Ibaraki 305-0044, Japan \\ ${ }^{4}$ Research Center for Knowledge Communities, University of Tsukuba, 1-2 Kasuga, Tsukuba, Ibaraki 305-8550, Japan
}

(Received 30 June 2014; published 11 September 2014)

\begin{abstract}
We demonstrate that silicon-vacancy (SiV) centers in diamond can be used to efficiently generate coherent optical photons with excellent spectral properties. We show that these features are due to the inversion symmetry associated with $\mathrm{SiV}$ centers. The generation of indistinguishable single photons from separated emitters at $5 \mathrm{~K}$ is demonstrated in a Hong-Ou-Mandel interference experiment. Prospects for realizing efficient quantum network nodes using $\mathrm{SiV}$ centers are discussed.
\end{abstract}

The realization of quantum networks, in which local quantum processing nodes are connected over long distances via optical photons, is an outstanding challenge in quantum information science [1]. Over the past few years, atomlike systems in the solid state have emerged as a promising platform for achieving this goal. Key building blocks have been demonstrated using nitrogen-vacancy (NV) centers in diamond, including long-lived qubit memory [2], spin-photon [3], and spin-spin entanglement [4], as well as teleportation between distant stationary qubits [5]. While NV centers can be used as excellent quantum registers, the current efforts to scale up these proof-of-concept experiments are limited by the small probability of coherent photon emission from NV centers and their spectral stability [6,7]. Here we demonstrate that silicon-vacancy $(\mathrm{SiV})$ centers in diamond can be used to efficiently generate coherent optical photons with excellent spectral stability. We show that these features are due to the inversion symmetry associated with $\mathrm{SiV}$ centers and demonstrate generation of indistinguishable single photons from separate emitters in a Hong-Ou-Mandel (HOM) interference experiment [8].

The negatively charged $\mathrm{SiV}$ center in diamond consists of a silicon atom and a split vacancy, as shown in Fig. 1(a) $[9,10]$. The silicon atom is centered between two empty lattice sites, and this $D_{3 \mathrm{~d}}$ geometry forms an inversion symmetric potential for the electronic orbitals [9]. Recent measurements [10,11] and first principle calculations [12] have contributed to a detailed understanding of the electronic structure of the $\mathrm{SiV}$ center. As shown in Fig. 1(b), the ground and excited states each consist of a fourfold degenerate manifold where two degenerate orbitals are

Published by the American Physical Society under the terms of the Creative Commons Attribution 3.0 License. Further distribution of this work must maintain attribution to the author(s) and the published article's title, journal citation, and DOI. occupied by an $S=1 / 2$ particle [13]. At zero magnetic field, the degeneracy is partially lifted by the spin-orbit interaction. Each excited state has dipole transitions to the two ground states forming an optical $\Lambda$ system, resulting in the emission spectrum shown in Fig. 1(c). These four transitions comprise the zero-phonon line (ZPL), which contains more than $70 \%$ of the total fluorescence. Remarkably, as discussed below, the inversion symmetry (a)
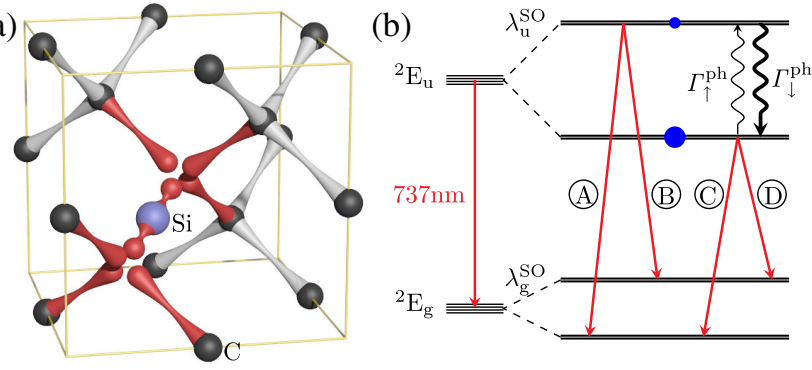

(c)

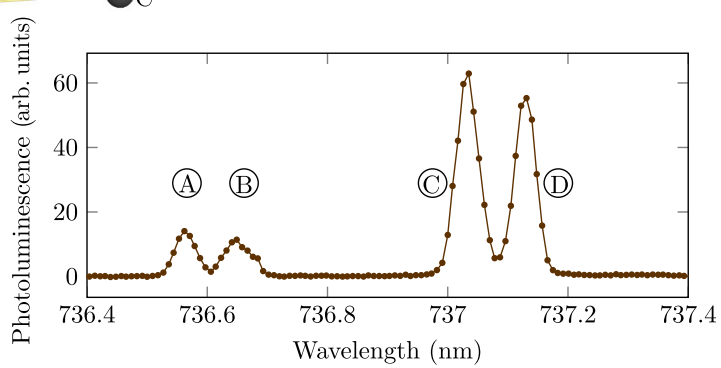

FIG. 1 (color online). Electronic structure and optical transitions of the SiV center. (a) The center is aligned along a $\langle 111\rangle$ axis of the diamond host crystal, with the silicon atom (Si) located in the middle of two empty lattice sites. The system has $D_{3 \mathrm{~d}}$ symmetry which includes inversion symmetry. (b) The optical transition is between different parity states, ${ }^{2} E_{u}$ and ${ }^{2} E_{g}$. Spin orbit interaction $\left(\lambda_{u}^{\mathrm{SO}} \sim 250 \mathrm{GHz}, \lambda_{g}^{\mathrm{SO}} \sim 50 \mathrm{GHz}\right)$ partially lifts the degeneracy giving rise to doublets in the ground and excited states. Transitions $A, B, C, D$ are all dipole allowed. (c) The emission spectrum measured using off-resonant excitation at $532 \mathrm{~nm}$ on a single $\mathrm{SiV}$ center at $4.5 \mathrm{~K}$. 
results in weak coupling of the ZPL transitions to charge fluctuations in the $\mathrm{SiV}$ environment. This leads to the absence of spectral diffusion [14] and a narrow inhomogeneous distribution [15].

To demonstrate coherent emission of indistinguishable single photons from separate $\mathrm{SiV}$ centers, we use a HOM interference experiment. The interference of two identical single photons impinging on a beam splitter results in perfect photon bunching, with a vanishing probability of detecting coincident photons at the two different output ports. In our experiments, two separate $\mathrm{SiV}$ centers, cooled to cryogenic temperatures, were excited using a twochannel confocal optical microscope shown in Fig. 2(a). Dichroic mirrors were used to simultaneously collect the $\mathrm{SiV}$ fluorescence on both the ZPL $(\lambda \sim 737 \mathrm{~nm})$ and phonon-side-band (PSB, $\lambda \sim 760-860 \mathrm{~nm}$ ). In order to isolate a single two-level transition, the emission spectrum was filtered by solid etalons [Fig. 2(b)] with a free spectral range of $20 \mathrm{GHz}$ and a bandwidth of $1 \mathrm{GHz}$. The etalons were tuned by temperature to transition $C$, and
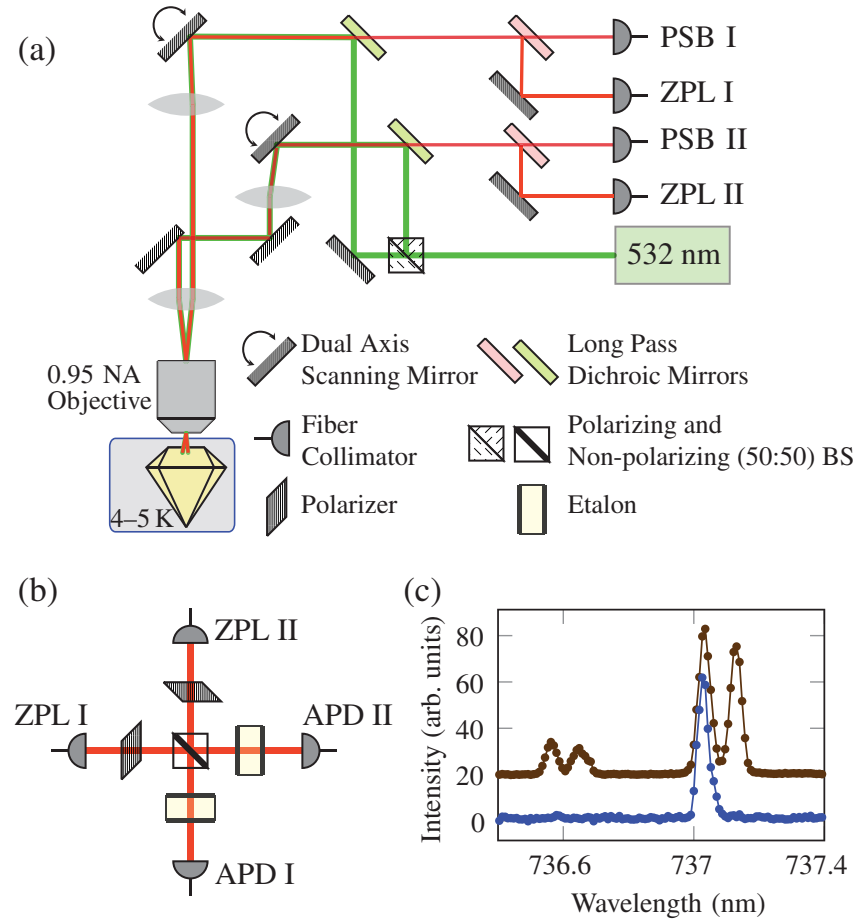

FIG. 2 (color online). Schematic of the two-channel confocal microscope built for the HOM experiment. (a) Channels I and II were used to address different emitters separated by tens of micrometers in the same sample. A continuous-wave 532-nm laser was used for excitation, and fluorescence was collected in single-mode fibers on ZPL and PSB ports simultaneously. (b) Collected ZPL fluorescence from the two channels were directed onto a free-space 50:50 nonpolarizing beam splitter. Linear polarizers were used to control the polarization of the single photons varying their distinguishability. Etalons were used to filter transition $C$ before detection. (c) Emission spectrum before (brown) and after the etalons (blue). the transmitted fluorescence spectrum is shown in Fig. 2(c), where only a single peak is visible as desired for indistinguishable photon generation.

To probe the inhomogeneous distribution (see the Supplemental Material [16]) within the sample and select spectrally overlapping sites, the emitters were resonantly excited with a 737-nm probe laser using the ZPL. The laser was tuned to the center frequency $\left(\nu_{0}\right)$ of the inhomogeneous distribution for transition $C$ while monitoring fluorescence intensity in the PSB. Figure 3(a) shows the diamond sample imaged by this technique in a region where the resonant site density was high, leading to a high background in any photon correlation experiments. In order to isolate single $\mathrm{SiV}$ centers and minimize background from other emitters [22], the laser was tuned to the edge of the inhomogeneous distribution $\left(\nu_{1}\right)$ in Fig. 3(b). Figures 3(c) and 3(d) show the two emitters that were chosen for the HOM experiment at frequency $\nu \sim \nu_{1}$. The images in Figures 3(c) and 3(d) were taken under 532-nm excitation while detecting ZPL photons through the etalons. Here the frequency selectivity is limited by the bandwidth of the etalons $(\sim 1 \mathrm{GHz})$, and therefore, more emitters are visible than in the resonant excitation scan [Fig. 3(b)]. Photoluminescence excitation (PLE) spectra of the emitters, $\mathrm{SiV}_{\mathrm{I}}$ (green) and $\mathrm{SiV}_{\mathrm{II}}$ (pink), reveal transitions separated by $52.1 \mathrm{MHz}$ with full width half maximum (FWHM) of 135.8 and $134.6 \mathrm{MHz}$, respectively. For comparison, the lifetime of the excited states was measured to be $1.73 \pm 0.05 \mathrm{~ns}$ at temperatures below $50 \mathrm{~K}$ corresponding to a transform limited linewidth of $94 \mathrm{MHz}$.

For the HOM measurement, single photons emitted from $\mathrm{SiV}_{\mathrm{I}}$ and $\mathrm{SiV}_{\mathrm{II}}$ on transition $C$ were directed to the input ports 1 and 2 of the beam splitter, respectively [see Figs. 2(a) and 2(b)]. Figure 4 shows two measurements where the degree of indistinguishability of single photons is varied by changing the photon polarization. The two data sets show the second-order intensity correlation function, $g^{2}(\tau)$, measured for indistinguishable (pink) and distinguishable (green) photon states. For identically polarized indistinguishable photons, we find $g_{\|}^{2}(0)=0.26 \pm 0.05$ where the error bars denote shot noise estimates. After rotating the fluorescence polarization of $\mathrm{SiV}_{\mathrm{II}}$ by 90 degrees to make the photon sources distinguishable, $g_{\perp}^{2}(0)=0.66 \pm 0.08$ was observed. These results clearly demonstrate two-photon interference corresponding to a measured HOM visibility of

$$
\eta=\frac{g_{\perp}^{2}(0)}{g_{\|}^{2}(0)+g_{\perp}^{2}(0)}=0.72 \pm 0.05
$$

The time dynamics of $g^{2}(\tau)$ is understood via independent measurements of the excited state lifetime, absorption line width, and detector timing response. Our model (solid curves, see the Supplemental Material [16]) is in excellent agreement with the measured time dynamics, showing that 
(a)

(b)

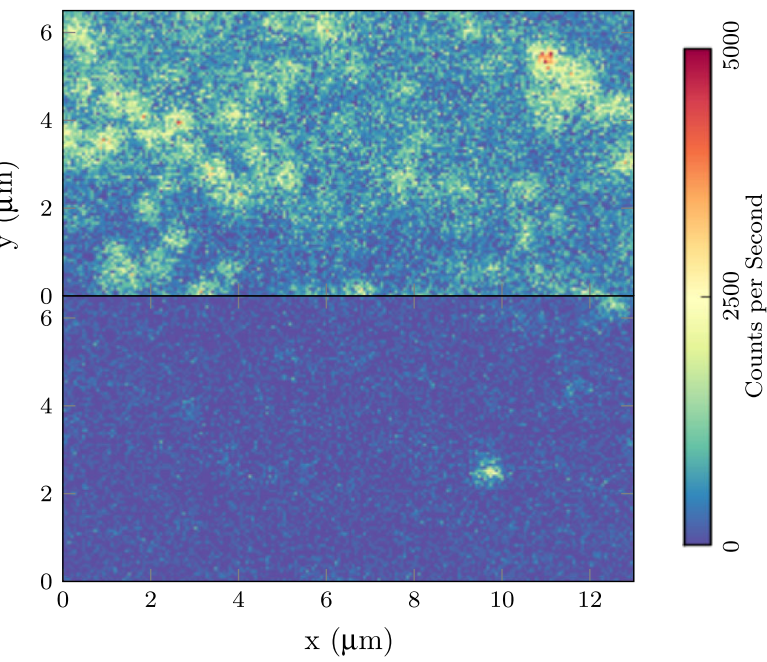

(c)

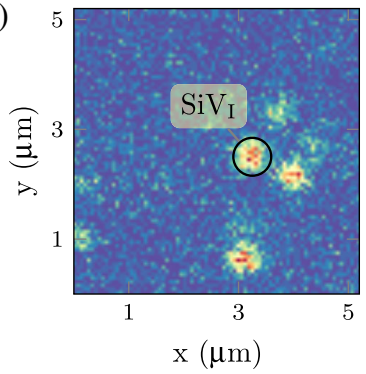

(d)

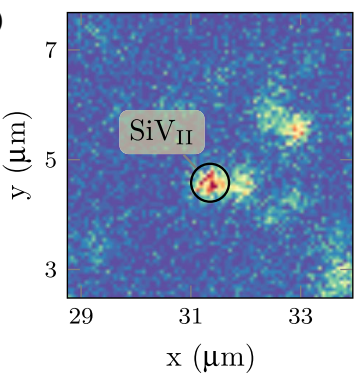

(e)

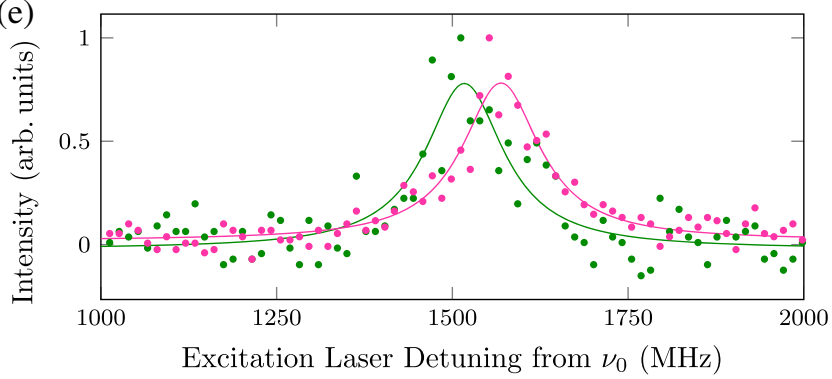

FIG. 3 (color online). Inhomogeneous distribution of $\mathrm{SiV}$ centers. (a) The probe laser frequency was fixed to the ensemble average of $\nu_{0}=406.7001 \mathrm{THz}$ for transition $C$ while scanning the sample. A high density of resonant emitters is visible with a large background. The color bar applies to (a)-(d). (b) Scan of the same region with the laser tuned to $\nu_{1}=\nu_{0}+1.5 \mathrm{GHz}$. Because of the narrow inhomogeneous distribution, only few resonant sites are visible, and the background level is low. (c) and (d) show the two emitters, $\mathrm{SiV}_{\mathrm{I}}$ and $\mathrm{SiV}_{\text {II }}$, used for the HOM interference experiment at frequency $\nu \sim \nu_{1}$. (e) PLE spectrum for $\mathrm{SiV}_{\mathrm{I}}$ (green) and $\mathrm{SiV}_{\mathrm{II}}$ (pink) with measured FWHM of 135.8 and 134.6 MHz, respectively, and lines separated by $52.1 \mathrm{MHz}$.

the emitters were spectrally stable throughout the 4-hour acquisition period. We find that the interference visibility, $\eta$, is limited by about equal contributions from detector timing response and background events.

We next turn to a discussion of the key properties of $\mathrm{SiV}$ centers which made the present observations possible. Despite uncertainty about the absolute quantum yield [14], the strong ZPL of $\mathrm{SiV}$ [23] means that photons are

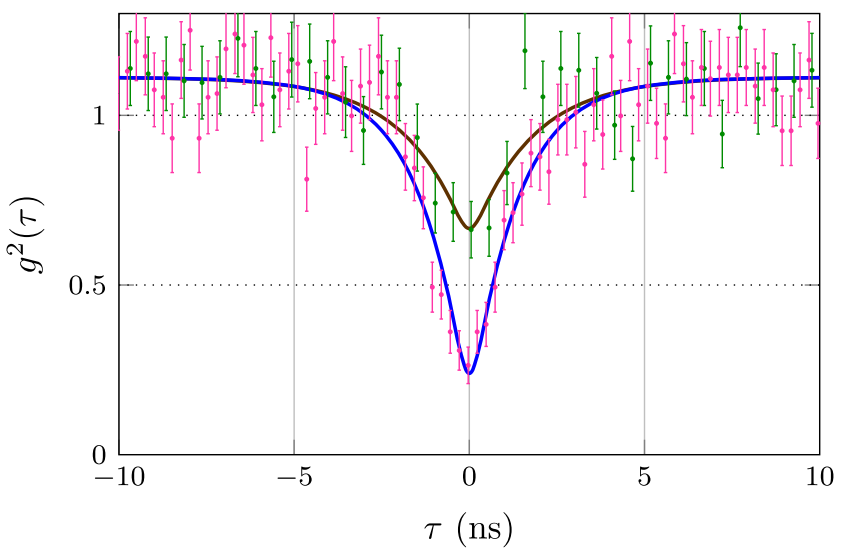

FIG. 4 (color online). HOM interference experiment. The second-order intensity correlation function $g^{2}(\tau)$ is plotted for two cases: (i) pink data show the results for indistinguishable single photons with identical polarizations, $g_{\|}^{2}(0)=0.26 \pm 0.05$. The error bars denote shot noise estimates. (ii) Green data show the results when photons from one emitter are orthogonally polarized and hence distinguishable, $g_{\perp}^{2}(0)=0.66 \pm 0.08$. The blue and brown solid lines represent our model using independently measured parameters, only fitting a single parameter for background events in both datasets.

emitted at high rates into the optical transition of interest. Inhomogeneous broadening corresponded to only a few transition line widths (see the Supplemental Material [16]), and high spectral stability of the transitions has been observed in bulk diamond [14] and nanodiamonds [13]. Together with these observations, our work shows that the optical coherence properties of $\mathrm{SiV}$ centers can be superior to those of NV centers [7,24]. Some of this advantage can be understood to result from the inversion symmetry of $\mathrm{SiV}$ centers which reduces sensitivity to electric field. In addition, it is important to consider the effects of phonons (strain) resulting in homogenous (inhomogeneous) broadening mechanisms.

The electronic orbitals of the $\mathrm{SiV}$ center are parity eigenstates due to the inversion symmetry of the defect. The optical transitions take place between states of different parity, ${ }^{2} E_{g}$ and ${ }^{2} E_{u}$, which differ in phase but have similar charge densities [12]. This small change in the electronic charge density results in the strong ZPL since optical excitations do not couple efficiently to local vibrations. The coherence of the optical transitions can also suffer from spectral diffusion, a time-dependent change in the optical transition frequencies that results in an increased line width. This effect is commonly observed for NV centers, where the dominant source of spectral diffusion has been shown to be from local electronic charge fluctuations [25]. These changes in the charge environment result in a fluctuating electric field at the emitter that reduces the coherence of the optical transitions via dc Stark shift $[7,26]$. The sensitivity of the optical transition frequencies to electric field fluctuations depends on the permanent electric dipole moments 
of the orbital states of the emitter. Since the electronic states of the $\mathrm{SiV}$ center have vanishing permanent electric dipole moments due to their inversion symmetry, the optical transitions are relatively insensitive to external electric fields. This protects the optical coherence from charge dynamics in the crystal, preventing spectral diffusion and narrowing the inhomogeneous distribution of transition frequencies.

Additional homogeneous and inhomogeneous broadening mechanisms are provided by phonons and strain. Displacements of atoms in the host crystal can affect the optical transitions in two different ways. Static distortions, or strain, may reduce the symmetry of the defect and change the energy splittings [15] [shown in Fig. 1(b)]. A variation in local strain contributes to the inhomogeneous distribution of the resonance frequencies [16]. Displacements of the atoms can also give rise to dynamic effects during an optical excitation cycle. Acoustic phonons have been shown to cause orbital relaxation between $E_{X}$ and $E_{Y}$ states for the NV center in diamond [27]. For SiV centers, a similar process can happen between excited state orbitals by absorption $\left(\Gamma_{\uparrow}^{\mathrm{ph}}\right)$ or emission $\left(\Gamma_{\downarrow}^{\mathrm{ph}}\right)$ of an acoustic phonon, as shown in Fig. 1(b). Populations in the upper and lower excited state branches follow a Boltzmann distribution confirming thermalization of orbital states by phonons $[14,15]$. At low temperatures $\left(k_{B} T \ll \hbar \lambda_{\mathrm{u}}^{\mathrm{SO}} \sim 250 \mathrm{GHz}\right)$, spontaneous emission dominates over stimulated processes $\left(\Gamma_{\uparrow}^{\mathrm{ph}} \ll \Gamma_{\downarrow}^{\mathrm{ph}}\right)$. To obtain an optical transition isolated from the phonon bath, our experiments were performed at 4.5-5 K ( 100 GHz) using the lower excited state branch. At these temperatures, we estimate a thermal broadening on transition $C$ of about $12 \mathrm{MHz}$ [14].

Our observations establish the $\mathrm{SiV}$ center as an excellent source of indistinguishable single photons. A strong ZPL transition, narrow inhomogeneous distribution, and spectral stability combine to make it a promising platform for applications in the fields of quantum networks and longdistance quantum communication. In particular, it should be possible to integrate $\mathrm{SiV}$ centers inside nanophotonic cavities $[6,28-31]$ while maintaining their spectral properties owing to their insensitivity to electric fields. This may allow the realization of $\mathrm{GHz}$ bandwidth deterministic single photon sources [32] and a broadband system for quantum nonlinear optics at the single-photon level [33]. The small inhomogeneous distribution also makes $\mathrm{SiV}$ centers promising candidates as sources of multiple indistinguishable photons for linear optics quantum computing [34]. Furthermore, the spin degree of freedom in the ground state [13] can potentially be utilized to store quantum information, allowing the use of $\mathrm{SiV}$ centers as quantum registers for quantum network applications [35]. Coupling to the ${ }^{29} \mathrm{Si}$ nuclear spin via hyperfine interactions [36] might allow realization of long-lived quantum memories [2]. Beyond these specific applications, the symmetry arguments presented above suggest that inversion symmetry might play an important role in the identification of new centers with suitable properties for quantum information science and technology [37].

A. S. and K. J. contributed equally to this work.

We thank J. D. Thompson, N. de Leon, A. Gali, M. L. Goldman, and T. Zibrova for theoretical discussions and experimental help. This work was supported by NSF, CUA, ERC, EU projects (SIQS, DIADEMS, EQUAM), DFG (FOR 1482, FOR 1493, and SFBTR 21), JST, JSPS KAKENHI (No. 26246001), BMBF, and the Volkswagen Foundation for funding.

*sipahigil@physics.harvard.edu

[1] H. J. Kimble, Nature (London) 453, 1023 (2008).

[2] P. C. Maurer, G. Kucsko, C. Latta, L. Jiang, N. Y. Yao, S. D. Bennett, F. Pastawski, D. Hunger, N. Chisholm, M. Markham et al., Science 336, 1283 (2012).

[3] E. Togan, Y. Chu, A. S. Trifonov, L. Jiang, J. Maze, L. Childress, M. G. Dutt, A. S. Sørensen, P. Hemmer, A. Zibrov et al., Nature (London) 466, 730 (2010).

[4] H. Bernien, B. Hensen, W. Pfaff, G. Koolstra, M. Blok, L. Robledo, T. Taminiau, M. Markham, D. Twitchen, L. Childress et al., Nature (London) 497, 86 (2013).

[5] W. Pfaff, B. Hensen, H. Bernien, S. B. van Dam, M. Blok, T. H. Taminiau, M. J. Tiggelman, R. N. Schouten, M. Markham, D. J. Twitchen et al., Science 345, 532 (2014).

[6] A. Faraon, C. Santori, Z. Huang, V. M. Acosta, and R. G. Beausoleil, Phys. Rev. Lett. 109, 033604 (2012).

[7] Y. Chu, N. de Leon, B. Shields, B. Hausmann, R. Evans, E. Togan, M. J. Burek, M. Markham, A. Stacey, A. Zibrov et al., Nano Lett. 14, 1982 (2014).

[8] C. K. Hong, Z. Y. Ou, and L. Mandel, Phys. Rev. Lett. 59, 2044 (1987).

[9] J. P. Goss, R. Jones, S. J. Breuer, P. R. Briddon, and S. Öberg, Phys. Rev. Lett. 77, 3041 (1996).

[10] L. J. Rogers, K. D. Jahnke, M. W. Doherty, A. Dietrich, L. P. McGuinness, C. Müller, T. Teraji, H. Sumiya, J. Isoya, N. B. Manson, and F. Jelezko, Phys. Rev. B 89, 235101 (2014).

[11] C. Hepp, T. Müller, V. Waselowski, J. N. Becker, B. Pingault, H. Sternschulte, D. Steinmüller-Nethl, A. Gali, J. R. Maze, M. Atatüre, and C. Becher, Phys. Rev. Lett. 112, 036405 (2014).

[12] A. Gali and J. R. Maze, Phys. Rev. B 88, 235205 (2013).

[13] T. Müller, C. Hepp, B. Pingault, E. Neu, S. Gsell, M. Schreck, H. Sternschulte, D. Steinmüller-Nethl, C. Becher, and M. Atatüre, Nat. Commun. 5, 3328 (2014).

[14] L. J. Rogers, K. D. Jahnke, L. Marseglia, C. Müller, B. Naydenov, H. Schauffert, C. Kranz, T. Teraji, J. Isoya, L. P. McGuinness et al., Nat. Commun. 5, 5739 (2014).

[15] H. Sternschulte, K. Thonke, R. Sauer, P. C. Münzinger, and P. Michler, Phys. Rev. B 50, 14554 (1994).

[16] See the Supplemental Material, which includes Refs. [17-21], at http://link.aps.org/supplemental/10.1103/ PhysRevLett.113.113602 for more information on the sample, the inhomogeneous distribution, and the model used for the HOM experiment. 
[17] T. Teraji, T. Taniguchi, S. Koizumi, K. Watanabe, M. Liao, Y. Koide, and J. Isoya, Jpn. J. Appl. Phys. 51, 090104 (2012).

[18] R. Lettow, Y. L. A. Rezus, A. Renn, G. Zumofen, E. Ikonen, S. Götzinger, and V. Sandoghdar, Phys. Rev. Lett. 104, 123605 (2010).

[19] R. B. Patel, A. J. Bennett, I. Farrer, C. A. Nicoll, D. A. Ritchie, and A. J. Shields, Nat. Photonics 4, 632 (2010).

[20] E. B. Flagg, A. Muller, S. V. Polyakov, A. Ling, A. Migdall, and G. S. Solomon, Phys. Rev. Lett. 104, 137401 (2010).

[21] E. Neu, M. Agio, and C. Becher, Opt. Express 20, 19956 (2012).

[22] W. E. Moerner and L. Kador, Phys. Rev. Lett. 62, 2535 (1989).

[23] E. Neu, D. Steinmetz, J. Riedrich-Möller, S. Gsell, M. Fischer, M. Schreck, and C. Becher, New J. Phys. 13, 025012 (2011).

[24] A. Sipahigil, M. L. Goldman, E. Togan, Y. Chu, M. Markham, D. J. Twitchen, A. S. Zibrov, A. Kubanek, and M. D. Lukin, Phys. Rev. Lett. 108, 143601 (2012).

[25] P. Siyushev, H. Pinto, M. Vörös, A. Gali, F. Jelezko, and J. Wrachtrup, Phys. Rev. Lett. 110, 167402 (2013).

[26] P. Tamarat, T. Gaebel, J. Rabeau, M. Khan, A. Greentree, H. Wilson, L. Hollenberg, S. Prawer, P. Hemmer, F. Jelezko et al., Phys. Rev. Lett. 97, 083002 (2006).
[27] C. Kai-Mei Fu, C. Santori, P. E. Barclay, L. J. Rogers, N. B. Manson, and R. G. Beausoleil, Phys. Rev. Lett. 103, 256404 (2009).

[28] M. J. Burek, N. P. de Leon, B. J. Shields, B. J. Hausmann, Y. Chu, Q. Quan, A. S. Zibrov, H. Park, M. D. Lukin, and M. Lončar, Nano Lett. 12, 6084 (2012).

[29] B. J. Hausmann, B. J. Shields, Q. Quan, Y. Chu, N. P. de Leon, R. Evans, M. J. Burek, A. S. Zibrov, M. Markham, D. J. Twitchen et al., Nano Lett. 13, 5791 (2013).

[30] J. Riedrich-Möller, L. Kipfstuhl, C. Hepp, E. Neu, C. Pauly, F. Mücklich, A. Baur, M. Wandt, S. Wolff, M. Fischer et al., Nat. Nanotechnol. 7, 69 (2012).

[31] J. C. Lee, I. Aharonovich, A. P. Magyar, F. Rol, and E. L. $\mathrm{Hu}$, Opt. Express 20, 8891 (2012).

[32] A. Kuhn, M. Hennrich, and G. Rempe, Phys. Rev. Lett. 89, 067901 (2002).

[33] T. Tiecke, J. Thompson, N. de Leon, L. Liu, V. Vuletić, and M. Lukin, Nature (London) 508, 241 (2014).

[34] P. Kok, W. J. Munro, K. Nemoto, T. C. Ralph, J. P. Dowling, and G. Milburn, Rev. Mod. Phys. 79, 135 (2007).

[35] L. I. Childress, J. M. Taylor, A. Sørensen, and M. D. Lukin, Phys. Rev. A 72, 052330 (2005).

[36] A. M. Edmonds, M. E. Newton, P. M. Martineau, D. J. Twitchen, and S. D. Williams, Phys. Rev. B 77, 245205 (2008).

[37] J. Weber, W. Koehl, J. Varley, A. Janotti, B. Buckley, C. Van de Walle, and D. D. Awschalom, Proc. Natl. Acad. Sci. U.S.A. 107, 8513 (2010). 